\title{
Time Headway Requirements for String Stability of Homogeneous Linear Unidirectionally Connected Systems
}

\author{
Steffi Klinge and Richard H. Middleton \\ Hamilton Institute, NUI Maynooth \\ Maynooth, Co. Kildare, Ireland \\ Email: mail@steffi-klinge.de and Richard.Middleton@nuim.ie
}

\begin{abstract}
This paper investigates string stability issues in homogeneous strings of strictly proper feedback control systems with unidirectional nearest neighbour communications, using only linear systems with two integrators in the loop. We show under which conditions the induced $L_{2}$-norm of the disturbance to error transfer function is bounded independently of the string length when using a constant time headway and derive a formula for the infimal time headway to guarantee string stability.
\end{abstract}

\section{INTRODUCTION}

One control objective in the field of coordinated systems is formation control. In formation control a group of vehicles should follow a given group trajectory and in addition every vehicle needs to maintain a prescribed distance to the surrounding vehicles. Increasing commercial and private vehicle traffic motivates a growing interest in the one dimensional version of this problem which is often called 'platooning'. In this case we focus on a linear string of automobiles driving in a column.

In its simplest form platoon control requires a constant distance between the vehicles and the lead vehicle follows a given trajectory, e.g. [1]-[5]. To simplify communication requirements we consider the case where the automobiles are equipped with a local controller based on sensing the distance to the preceding vehicle. We call the string homogeneous if the dynamics of the vehicle and controller are independent of location in the string.

If every controller only uses the information of the separation to its predecessor the system structure will be triangular. Hence, studying the stability of the system is relatively easy. In other words, for a fixed string length, and appropriately designed local controllers, asymptotic and input-output stability can be guaranteed. Unfortunately, in some cases, these forms of stability are not uniform with respect to string length, and as the string length grows, the disturbance response may grow without bound. This effect is referred to as 'string instability'.

In the past, different definitions of string stability have been utilised. While most researchers work with input-output formulations, definitions involving the initial conditions and state space formulations can also be found, [6]. Due to the ease of working with the Euclidean norm [7], [8], it is often preferred to the maximum norm, [9].

The authors would like to thank the Science Foundation of Ireland for supporting this work with grant 07/RPR/I177.
It has been shown that it is not possible to achieve string stability in a homogeneous string of strictly proper feedback control systems with nearest neighbour communications when using only linear systems with two integrators in the open loop and constant inter-vehicle spacing, [3], [10]. This result is independent of the particular linear controller design, [7], [10]. The problem was also studied using partial differential equations, [11], [12] from the perspective of the slowest closed loop eigenvalue for problems with bidirectional control. However, string stability can be guaranteed with a speed dependent inter-vehicle spacing policy (also called 'time headway policy'), [13]. Other research was done on heterogeneous strings, i.e. the particular controller depends on the position within the string, [8], [14] and on nonlinear spacing policies, [15].

We would like to present a precise discussion of string stability of a homogeneous system with two integrators in the open loop of the subsystem and unidirectional nearest neighbour communication. First we will clarify the notation used and derive the disturbance-to-error-transfer function in Section II. Thereafter we will show that string instability can be avoided using a time headway policy only if the time headway is sufficiently large. In particular, we derive a formula for the infimal time headway to guarantee $L_{2}$-string stability in Section III. In Section IV string stability in the $L_{2}$ sense will be proved using a sufficiently large time headway. Examples in Section V illustrate the results.

\section{PRELIMINARIES}

We wish to discuss the stability of a simple chain of $N$ vehicles where all but the first should keep a fixed distance $x_{\mathrm{d}}$ to their predecessor. The first car follows a given trajectory. We will choose the same vehicle model with transfer function $P(s)$ and the same linear controller $C(s)$ for every subsystem, i.e. every car. The open loop transfer function $L(s)$ has exactly two poles at the origin, $L(s)=$ $P(s) C(s)=\frac{1}{s^{2}} \tilde{L}(s)$ with $\tilde{L}(0) \neq 0$. The position of the $i$ th vehicle $x_{i}$ depends on the disturbance $d_{i}$ and the actuator signal of the $i$ th controller $u_{i}$. The local control objective is to force the separation error $e_{i}$ to zero. Measurement noise is neglected for simplicity.

$$
\begin{aligned}
& x_{i}=P(s)\left(u_{i}+d_{i}\right) \\
& u_{i}=C(s) e_{i} \\
& e_{i}=x_{i-1}-x_{i}-x_{\mathrm{d}}
\end{aligned}
$$




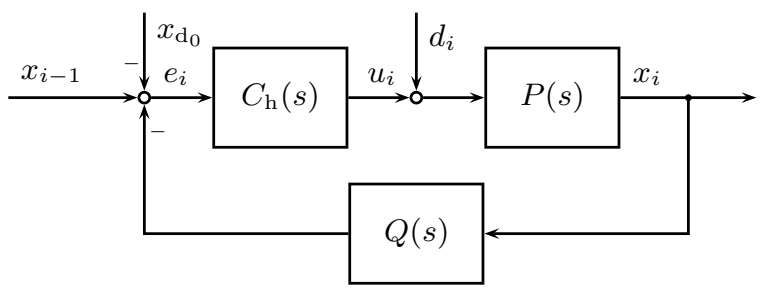

Fig. 1: Block diagram of the linear system with time headway

with the vector of error signals $\underline{e}(t)=\left(e_{1} e_{2} \cdots e_{N}\right)^{\mathrm{T}}$ and the disturbances $\underline{d}(t)=\left(\begin{array}{llll}d_{1} & d_{2} & \cdots & d_{N}\end{array}\right)^{\mathrm{T}}$. It is known that the absolute value of the complementary sensitivity function of a single subsystem, $T(s)=\frac{L(s)}{1+L(s)}$, is greater than one for a range of frequencies $\omega \in\left(\omega_{-}, \omega_{+}\right)$, and that the system therefore will be 'string unstable' for constant spacing $\left(x_{\mathrm{d}}=\right.$ const $)$, [3], [7].

We consider the following definition of $L_{2}$-string stability:

Definition 1 ( $L_{2}$-String Stability): Consider a string of $N$ dynamic systems with the local error signal $e_{i}$ and the disturbance $d_{i}$. The error signals $\underline{e}(t)$ depend on the disturbances $\underline{d}(t)$ in the following manner:

$$
\underline{e}(t)=H_{\mathrm{e}, \mathrm{d}}(s) * \underline{d}(t)
$$

where $\underline{e}, \underline{d} \in \mathbb{R}^{N}, N \in \mathbb{N}$ and $H_{\mathrm{e}, \mathrm{d}}(s): \mathbb{R}^{N} \rightarrow \mathbb{R}^{N}$. The system (4) is $L_{2}$-string stable if given any $\epsilon>0$ there exists a $\delta>0$ such that

$$
\|\underline{d}(\cdot)\|_{\mathrm{i}_{2}}<\delta \Rightarrow\|\underline{e}(\cdot)\|_{\mathrm{i}_{2}}<\epsilon
$$

where $\delta$ is independent of the string length $N$.

Since using a constant spacing policy the system is string unstable, a linear time headway $h$ is incorporated in the feedback path. In addition to a fixed vehicle separation, a velocity $v_{i}$ dependent distance is required between the vehicles, $x_{\mathrm{d}}=$ $x_{\mathrm{d}_{0}}+h v_{i}$. To simplify the following derivations and because we are interested in the disturbance to error behaviour we shall set $x_{\mathrm{d}_{0}}=0$ below. The complementary sensitivity function of the new subsystem (shown in Fig. 1) is $\Gamma(s)=$ $\frac{P(s) C_{\mathrm{h}}(s)}{1+P(s) C_{\mathrm{h}}(s) Q(s)}=\frac{1}{Q(s)} \frac{P(s) C(s)}{1+P(s) C(s)}$ with $C_{\mathrm{h}}(s)=\frac{C(s)}{Q(s)}$ and
$Q(s)=h s+1$.

Since the output of the $(i-1)$ th subsystem (position $x_{i-1}$ ) is the reference signal for the $i$ th system with the output $x_{i}$, we can write the transfer function $H_{\mathrm{x}_{i}, \mathrm{x}_{i-1}}(s)=\Gamma(s)$.

Consider a disturbance $d_{i}(s)$ that enters the $i$ th subsystem between the controller $C_{\mathrm{h}}(s)$ and the plant $P(s)$. It affects the output of the $i$ th subsystem with $H_{\mathrm{x}_{i}, \mathrm{~d}_{i}}(s)=C_{\mathrm{h}}^{-1}(s) \Gamma(s)$.

$$
x_{i}(s)=\Gamma(s) x_{i-1}(s)+C_{\mathrm{h}}^{-1}(s) \Gamma(s) d_{i}(s)
$$

The error signal $e_{i}$ for $2 \leq i \leq N$ can be expressed as

$$
\begin{aligned}
e_{i}(s)= & x_{i-1}(s)-Q(s) x_{i}(s) \\
= & \Gamma(s)\left(x_{i-2}(s)-Q(s) x_{i-1}(s)\right) \\
& +\Gamma(s) C_{\mathrm{h}}^{-1}(s)\left(d_{i-1}(s)-Q(s) d_{i}(s)\right) \\
= & \Gamma(s) e_{i-1}(s) \\
& +\Gamma(s) C_{\mathrm{h}}^{-1}(s)\left(d_{i-1}(s)-Q(s) d_{i}(s)\right)
\end{aligned}
$$

In vector form, we can write

$$
\begin{aligned}
& \underline{e}=\left[\begin{array}{cccc}
0 & & & 0 \\
\Gamma(s) & 0 & & \\
& \ddots & \ddots & \\
0 & & \Gamma(s) & 0
\end{array}\right] \underline{e} \\
& +\left[\begin{array}{cccc}
-Q(s) & & & 0 \\
1 & -Q(s) & & \\
& \ddots & \ddots & \\
0 & & 1 & -Q(s)
\end{array}\right] \Gamma(s) C_{\mathrm{h}}^{-1}(s) \underline{d} \\
& =\underbrace{\left[\begin{array}{cccc}
1 & & & 0 \\
-\Gamma(s) & 1 & & \\
& \ddots & \ddots & \\
0 & & -\Gamma(s) & 1
\end{array}\right]}_{\tilde{\Gamma}} \\
& \underbrace{\left[\begin{array}{cccc}
-1 & & & 0 \\
Q^{-1}(s) & -1 & & \\
& \ddots & \ddots & \\
0 & & Q^{-1}(s) & -1
\end{array}\right]}_{\tilde{Q}} \Gamma(s) C^{-1}(s) \underline{d}
\end{aligned}
$$

with $H_{\mathrm{e}, \mathrm{d}}=\tilde{\Gamma}^{-1} \tilde{Q} \Gamma(s) C^{-1}(s)$.

We wish to discus string stability according to Definition 1 . That is, we require $L_{2}$ bounded error signals independent of the string length $N$ for any vector of $L_{2}$ bounded disturbances. Thus, the induced $L_{2}$-norm of the operator $H_{\mathrm{e}, \mathrm{d}}$ must be bounded independently of $N$.

The induced $L_{2}$-norm of a matrix operator $A(j \omega)$ is the supremum over frequency of its largest singular value, $\sigma_{\max }$ :

$$
\|A(j \omega)\|_{i_{2}}=\operatorname{ess} \sup _{\omega \in \mathbb{R}} \sigma_{\max }(A(j \omega))=\operatorname{ess} \sup _{\omega \in \mathbb{R}} \sqrt{\lambda_{\max }\left(A^{*} A\right)}
$$

Where $\bar{A}$ is the complex conjugate of $A$ and $A^{*}$ its Hermitian adjoint with $\left(A^{*}\right)_{i, j}=(\bar{A})_{j, i}$.

\section{INDUCED NORM OF $H_{\mathrm{e}, \mathrm{d}}$ FOR $\|\Gamma\|>1$}

Lemma 1 (String instability for $\|\Gamma\|>1$ ): Suppose the disturbance to error performance of an interconnected system is described by (7), where $\Gamma(s)=\frac{1}{Q(s)} \frac{P(s) C(s)}{1+P(s) C(s)}$ and $Q(s)=h s+1$ and the controller $C(s)$ internally stabilises the plant $P(s)$. Suppose also that there exists a frequency $\omega_{0}$ such that $\left|\Gamma\left(j \omega_{0}\right)\right|>1$, then there exists a $\tau_{0}>0$ such that $\left\|H_{\mathrm{e}, \mathrm{d}}\right\|_{\mathrm{i}_{2}}=\left\|\tilde{\Gamma}^{-1} \tilde{Q} \Gamma(s) C^{-1}(s)\right\|_{\mathrm{i}_{2}} \geq\left|\Gamma\left(j \omega_{0}\right)\right|^{N} \tau_{0}$.

Proof: The over all disturbance-to-error-transfer function $H_{\mathrm{e}, \mathrm{d}}$ is

$$
\begin{aligned}
H_{\mathrm{e}, \mathrm{d}} & =\tilde{\Gamma}^{-1} \tilde{Q} \Gamma C^{-1} \\
& =\left[\begin{array}{cccc}
-1 & & & 0 \\
Q^{-1}-\Gamma & -1 & & \\
\Gamma\left(Q^{-1}-\Gamma\right) & Q^{-1}-\Gamma & -1 & \\
\vdots & \vdots & \ddots & \\
\Gamma^{N-2}\left(Q^{-1}-\Gamma\right) & \Gamma^{N-3}\left(Q^{-1}-\Gamma\right) & \cdots & -1
\end{array}\right] \Gamma C^{-1}
\end{aligned}
$$


Note that

$$
\begin{aligned}
\left\|H_{\mathrm{e}, \mathrm{d}}\right\|_{\mathrm{i}_{2}} & =\operatorname{ess} \sup _{\omega \in \mathbb{R}}|| H_{\mathrm{e}, \mathrm{d}}(j \omega) \|_{\mathrm{i}_{2}} \\
& \geq \operatorname{ess} \sup _{\omega \in \mathbb{R}} \max _{i, j}\left|\left(H_{\mathrm{e}, \mathrm{d}}\right)_{i j}\right| \\
& \geq \operatorname{ess} \sup _{\omega \in \mathbb{R}}\left|\Gamma^{N-2}\left(Q^{-1}-\Gamma\right) \Gamma C^{-1}\right| \\
& =\operatorname{ess} \sup _{\omega \in \mathbb{R}}|\Gamma|^{N-1}\left|\left(Q^{-1}-\Gamma\right)\right|\left|C^{-1}\right|
\end{aligned}
$$

The last equality holds because $\Gamma, Q$ and $C$ are scalar transfer functions. Under the assumption that there exists a non zero ${ }^{1}$ frequency $\omega_{0}$ for which $\left|\Gamma\left(j \omega_{0}\right)\right|>1$, [6], [9], the absolute value of $\left(Q^{-1}-\Gamma\right)$ and $C^{-1}$ cannot be zero at $\omega_{0}$ as we now demonstrate. First, suppose $C^{-1}\left(j \omega_{0}\right)=0$. So $C(s)$ has two poles at $s= \pm j \omega_{0}$. Since a marginally stable pole zero cancellation would contradict internal stability of the loop $P\left(j \omega_{0}\right)$ cannot be zero. Hence,

$$
\begin{aligned}
\Gamma\left(j \omega_{0}\right) & =\frac{1}{Q\left(j \omega_{0}\right)} \frac{P\left(j \omega_{0}\right) C\left(j \omega_{0}\right)}{1+P\left(j \omega_{0}\right) C\left(j \omega_{0}\right)} \\
& =\frac{1}{Q\left(j \omega_{0}\right)} \frac{1}{\frac{C^{-1}\left(j \omega_{0}\right)}{P\left(j \omega_{0}\right)}+1} \\
& =\frac{1}{Q\left(j \omega_{0}\right)}
\end{aligned}
$$

and thus $\left|\Gamma\left(j \omega_{0}\right)\right|=\left|Q^{-1}\left(j \omega_{0}\right)\right|<1$ which contradicts the first assumption that $\left|\Gamma\left(j \omega_{0}\right)\right|>1$. Also, the magnitude of $Q^{-1}\left(j \omega_{0}\right)-\Gamma\left(j \omega_{0}\right)$ cannot be zero because $\left|Q^{-1}\right|<1$ for all frequencies greater than zero and $\left|\Gamma\left(j \omega_{0}\right)\right|>1$.

Therefore the induced $L_{2}$-norm of $H_{e, d}$ will grow exponentially with the string length $N$ and the system will be string unstable with $\tau_{0}=\left|C^{-1}\left(j \omega_{0}\right)\right|\left|Q^{-1}\left(j \omega_{0}\right)-\Gamma\left(j \omega_{0}\right)\right|$.

Thus, one necessary condition for string stability is that $|\Gamma(j \omega)| \leq 1$ for all $\omega$. Note that

$$
|\Gamma(j \omega)|^{2}=\frac{1}{1+\omega^{2} h^{2}}\left|\frac{L(j \omega)}{1+L(j \omega)}\right|^{2} \leq 1 \quad \forall \omega
$$

Hence the infimal time headway essential to permit string stability (since otherwise $\|\Gamma\|_{\mathrm{i}_{2}}>1$ ) is $h_{0}$

$$
h_{0}:=\sqrt{\max _{\omega}\left(\frac{\left|\frac{L(j \omega)}{1+L(j \omega)}\right|^{2}-1}{\omega^{2}}\right)}
$$

Since the maximum in (12) can be attained at $\omega=0$ or at at least one $\omega_{0} \neq 0$, we will distinguish between two cases:

(a) The maximum in (12) is attained at $\omega=0$ only. Using L'Hôpital's Rule and the fact that $\tilde{L}(0)=\overline{\tilde{L}}(0)=|\tilde{L}(0)|$ condition (12) becomes

$$
h_{0}=\lim _{\omega \rightarrow 0} \sqrt{\frac{\left|\frac{L(j \omega)}{1+L(j \omega)}\right|^{2}-1}{\omega^{2}}}=\sqrt{2 /|\tilde{L}(0)|}
$$

Hence, choosing $h=\sqrt{2 /|\tilde{L}(0)|}$ guarantees that $|\Gamma| \leq 1$ and $|\Gamma|=1$ only at $\omega=0$. In fact, this condition has a simple geometric interpretation. For $h=\sqrt{2 /|\tilde{L}(0)|}$ the second derivative of $|\Gamma|$ at $\omega=0$ is zero, $\left.\frac{\mathrm{d}^{2}}{\mathrm{~d} \omega^{2}}|\Gamma(\omega)|\right|_{\omega=0}=0$. Since $|\Gamma|$ is equal to 1 at the origin, it would be greater than 1 for some frequency $\omega^{\prime}>0$ if its second derivative at the origin would be greater or equal to zero.

(b) The maximum in (12) is attained at at least one $\omega_{0} \neq 0$. In that case $|\Gamma| \leq 1$ and $|\Gamma|=1$ only at $\omega=0$ and $\omega=\omega_{0}$. Condition (12) becomes

$$
h_{0}=\frac{\sqrt{\left|\frac{L\left(j \omega_{0}\right)}{1+L\left(j \omega_{0}\right)}\right|^{2}-1}}{\omega_{0}}
$$

IV. INDUCED NORM OF $H_{\mathrm{e}, \mathrm{d}}$ FOR $|\Gamma| \leq 1$

As we have seen that string stability cannot be achieved for a system with a time headway less than $h_{0}$ we will now choose a time headway of $h>h_{0}$.

Lemma 2 (String stability for $h>h_{0}$ ): Suppose the disturbance to error performance of an interconnected system is described by (7), where $\Gamma(s)=\frac{1}{Q(s)} \frac{P(s) C(s)}{1+P(s) C(s)}$ and $Q(s)=h s+1$. Suppose the time headway $h$ is strictly greater than $h_{0}$ as defined in (12) and the controller $C(s)$ internally stabilises the plant $P(s)$. Then there exists a $\tau_{0}$ such that $\left\|H_{\mathrm{e}, \mathrm{d}}\right\|_{\mathrm{i}_{2}}=\left\|\tilde{\Gamma}^{-1} \tilde{Q} \Gamma(s) C^{-1}(s)\right\|_{\mathrm{i}_{2}} \leq \tau_{0}$.

Proof: Using the structure of $\tilde{\Gamma}$ and $\tilde{Q}$ we can write $H_{\mathrm{e}, \mathrm{d}}$ as

$$
\begin{aligned}
& H_{\mathrm{e}, \mathrm{d}}=\tilde{\Gamma}^{-1} \tilde{Q} \Gamma C^{-1}
\end{aligned}
$$

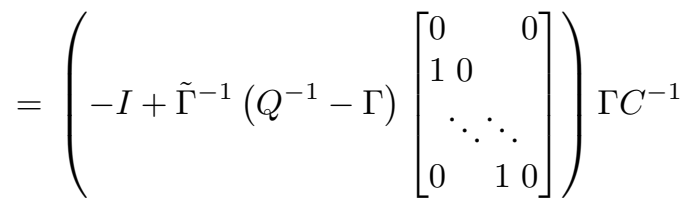

Using the triangle inequality we can bound the induced $L_{2}$ norm of $H_{\mathrm{e}, \mathrm{d}}$ as

$$
\left\|H_{\mathrm{e}, \mathrm{d}}\right\|_{\mathrm{i}_{2}} \leq\left(1+\left\|\tilde{\Gamma}^{-1}\left(Q^{-1}-\Gamma\right)\right\|_{\mathrm{i}_{2}}\right)|\Gamma|_{\mathrm{i}_{2}}\left|C^{-1}\right|_{\mathrm{i}_{2}}
$$

Since the norms of $\Gamma$ and $C^{-1}$ do not depend on the string length, the norm of $\tilde{\Gamma}^{-1}\left(Q^{-1}-\Gamma\right)$ can be used to bound $\left\|H_{\mathrm{e}, \mathrm{d}}\right\|_{\mathrm{i}_{2}}$.

$\left\|\tilde{\Gamma}^{-1}\left(Q^{-1}-\Gamma\right)\right\|_{\mathrm{i}_{2}}=\operatorname{ess} \sup _{\omega \in \mathbb{R}}\left(\left(\sigma_{\min }(\tilde{\Gamma})\right)^{-1}\left|Q^{-1}-\Gamma\right|\right)$

Using the Gersgorin-Theorem (see e.g. [16]), we can estimate the minimal Eigenvalue of a matrix.

$$
\begin{gathered}
\lambda_{\min }(A) \geq \max \left\{\min _{i}\left(a_{i i}-\sum_{j=1, j \neq i}^{n}\left|a_{i j}\right|\right),\right. \\
\left.\min _{j}\left(a_{j j}-\sum_{i=1, i \neq j}^{n}\left|a_{i j}\right|\right)\right\}
\end{gathered}
$$

\footnotetext{
${ }^{1}$ Since $\Gamma(0)=1, \omega_{0} \neq 0$.
} 
For $\tilde{\Gamma}^{*} \tilde{\Gamma}$ we obtain

$$
\begin{aligned}
\lambda_{\min }\left(\tilde{\Gamma}^{*} \tilde{\Gamma}\right) & \geq \min \left\{1+|\Gamma|^{2}-|\Gamma|, 1+|\Gamma|^{2}-2|\Gamma|, 1-|\Gamma|\right\} \\
& =1+|\Gamma|^{2}-2|\Gamma|=(1-|\Gamma|)^{2}
\end{aligned}
$$

Thus, the induced $L_{2}$-norm of $\tilde{\Gamma}^{-1}\left(Q^{-1}-\Gamma\right)$ can be bounded as

$$
\begin{aligned}
\left\|\tilde{\Gamma}^{-1}\left(Q^{-1}-\Gamma\right)\right\|_{\mathrm{i}_{2}} & \leq \operatorname{ess} \sup _{\omega \in \mathbb{R}} \frac{\left|Q^{-1}-\Gamma\right|}{1-|\Gamma|} \\
& =\operatorname{ess} \sup _{\omega \in \mathbb{R}} \frac{\left|Q^{-1}\right| \frac{1}{|1+L|}}{1-\left|Q^{-1}\right| \frac{|L|}{|1+L|}} \\
& =\operatorname{ess} \sup _{\omega \in \mathbb{R}} \frac{1}{|Q||1+L|-|L|}
\end{aligned}
$$

and from (16)

$$
\begin{aligned}
\left\|H_{\mathrm{e}, \mathrm{d}}\right\|_{\mathrm{i}_{2}} \leq & \left(1+\operatorname{ess} \sup _{\omega \in \mathbb{R}} \frac{1}{|Q \|| 1+L|-| L \mid}\right) \\
& \cdot \text { ess } \sup _{\omega \in \mathbb{R}}|\Gamma| \operatorname{ess} \sup _{\omega \in \mathbb{R}}\left|C^{-1}\right|
\end{aligned}
$$

since $|\Gamma|$ and $\left|C^{-1}\right|$ are bounded independently of the string length $N$. However, we need to have a closer look at (21) for $\omega=0$, where $|\Gamma(0)|=1$.

$$
\begin{aligned}
& \lim _{\omega \rightarrow 0} \frac{1}{|Q \|| 1+L|-| L \mid} \\
& =\lim _{\omega \rightarrow 0} \frac{1}{\sqrt{h^{2} \omega^{2}+1} \sqrt{1-\frac{1}{\omega^{2}}(\tilde{L}+\tilde{\tilde{L}})+\frac{1}{\omega^{4}}|\tilde{L}|^{2}}-\frac{1}{\omega^{2}}|\tilde{L}|} \\
& =\lim _{\omega \rightarrow 0} \frac{\omega^{2}}{\sqrt{h^{2} \omega^{2}+1} \sqrt{\omega^{4}-\omega^{2}(\tilde{L}+\overline{\tilde{L}})+|\tilde{L}|^{2}}-|\tilde{L}|}
\end{aligned}
$$

Using L'Hôpital's Rule, (22) becomes

$$
\begin{aligned}
& \lim _{\omega \rightarrow 0} \frac{1}{|Q||1+L|-|L|} \\
&= \lim _{\omega \rightarrow 0}\left(\frac{1}{2} \frac{h^{2}}{\sqrt{h^{2} \omega^{2}+1}} \sqrt{\omega^{4}-\omega^{2}(\tilde{L}+\overline{\tilde{L}})+|\tilde{L}|^{2}}\right. \\
&+\sqrt{h^{2} \omega^{2}+1} \\
& \cdot \frac{1}{2} \frac{2 \omega^{2}-(\tilde{L}+\overline{\tilde{L}})-\omega^{2} \frac{\mathrm{d}}{\mathrm{d} \omega^{2}}(\tilde{L}+\overline{\tilde{L}})+2|\tilde{L}| \frac{\mathrm{d}}{\mathrm{d} \omega^{2}}|\tilde{L}|}{\sqrt{\omega^{4}-\omega^{2}(\tilde{L}+\overline{\tilde{L}})+|\tilde{L}|^{2}}} \\
&=\left.\frac{\mathrm{d}}{\mathrm{d} \omega^{2}}|\tilde{L}|\right)^{-1} \\
& \frac{1}{2} h^{2}|\tilde{L}(0)|-\frac{1}{2} \frac{1}{\mid \tilde{L}(0) \tilde{\tilde{L}}(0)}
\end{aligned}
$$

At zero frequency $\tilde{L}(0)=\overline{\tilde{L}}(0)=|\tilde{L}(0)|$. Since $h$ is strictly greater than $h_{0}$ and therefore greater than $\sqrt{2 /|\tilde{L}(0)|}$, $\lim _{\omega \rightarrow 0}(|Q||1+L|-|L|)^{-1}$ is bounded. Hence, $\left\|H_{\mathrm{e}, \mathrm{d}}\right\|_{\mathrm{i}_{2}}$ is bounded independently of $N$ and the system is string stable according to Definition 1.
We have proven string stability for $h>h_{0}$, and string instability for $h<h_{0}$. It remains therefore to consider the case where $h=h_{0}$. We will show that the induced $L_{2}$-norm of $H_{\mathrm{e}, \mathrm{d}}$ will grow at least as fast as the square root of the string length $N$.

First, we will analyse case (b) where $h_{0}$ is chosen according to (14) and $\left|\Gamma\left(j \omega_{0}\right)\right|=1$. Since the first element of $H_{\mathrm{e}, \mathrm{d}}^{*} H_{\mathrm{e}, \mathrm{d}}$ is

$$
\left(H_{\mathrm{e}, \mathrm{d}}^{*} H_{\mathrm{e}, \mathrm{d}}\right)_{1,1}=\left(1+\sum_{i=0}^{N-2}|\Gamma|^{2^{i}}\left|Q^{-1}-\Gamma\right|^{2}\right)|\Gamma|^{2}\left|C^{-1}\right|^{2},
$$

$\left|Q^{-1}\left(j \omega_{0}\right)-\Gamma\left(j \omega_{0}\right)\right| \neq 0$, and $C^{-1}\left(j \omega_{0}\right) \neq 0$ the norm of $\left(H_{\mathrm{e}, \mathrm{d}}^{*} H_{\mathrm{e}, \mathrm{d}}\right)_{1,1}$ will grow with the string length $N$. Hence the largest Eigenvalue of $H_{\mathrm{e}, \mathrm{d}}^{*} H_{\mathrm{e}, \mathrm{d}}$ and therefore the square of the induced $L_{2}$-norm of $H_{\mathrm{e}, \mathrm{d}}$ will grow with the string length $N$.

The proof for case (a) is given in the appendix.

\section{EXAMPLES}

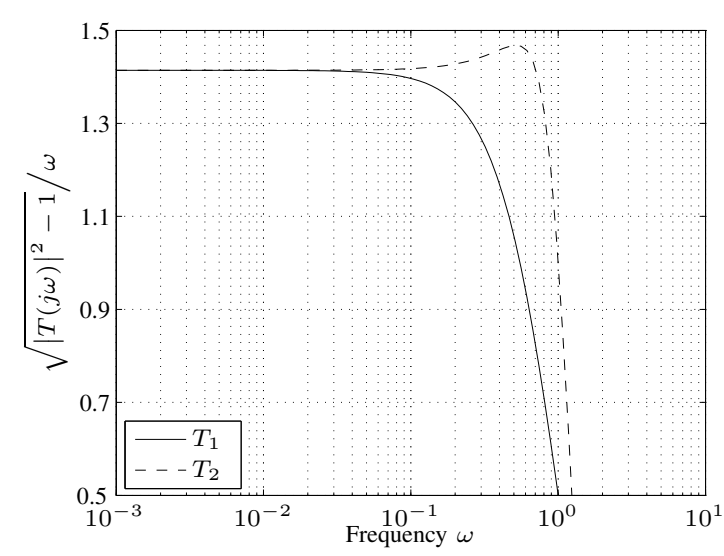

(a) $\sqrt{|T(j \omega)|^{2}-1} / \omega$ for different transfer functions

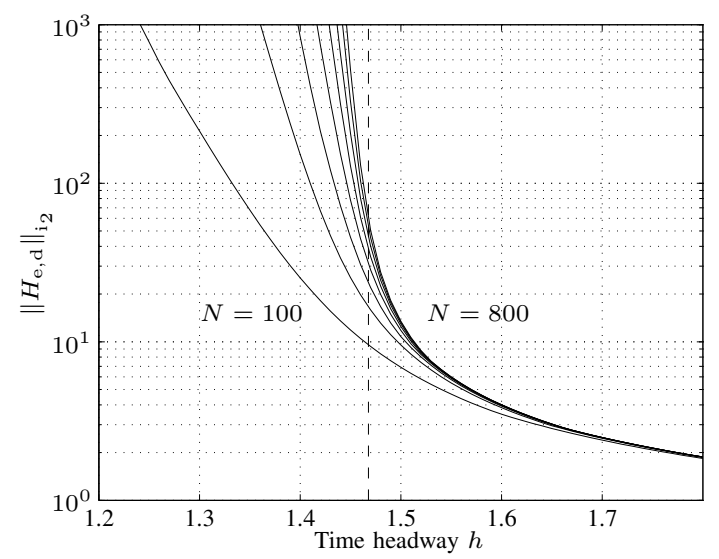

(b) Induced $L_{2}$-norm of $H_{\mathrm{e}, \mathrm{d}}$ for different time headways $\mathrm{h}$

Example 1 (Infimal Time Headway $h_{0}$ ): In order to find the infimal time headway $h_{0}$, the maximum over all frequencies of $\left(|T(j \omega)|^{2}-1\right) / \omega^{2}$ must be evaluated. For $T_{1}(s)=\frac{s+1}{s^{2}+s+1}$ the maximum is achieved at $\omega=0$ and 
$h_{0}=\sqrt{2}$ is chosen according to (13). For $T_{2}(s)=\frac{2 s+1}{s^{2}+2 s+1}$ it is achieved at $\omega=\omega_{0} \approx 0.5$. Thus, $h_{0} \approx 1.47$ is chosen according to (14). In Fig. 2a both cases are illustrated.

Example 2 (Induced $L_{2}$-Norm of $H_{\mathrm{e}, \mathrm{d}}$ ): Fig. $2 \mathrm{~b}$ shows $\left\|H_{\mathrm{e}, \mathrm{d}}\right\|_{\mathrm{i}_{2}}$ for different time headways $h$ and string lengths $N$. For time headways less than $h_{0}$ (dashed line) the induced $L_{2}$ norm of $H_{\mathrm{e}, \mathrm{d}}$ grows exponentially with the string length $N$. However, if the time headway is sufficiently large, $h>h_{0}$, $\left\|H_{\mathrm{e}, \mathrm{d}}\right\|_{\mathrm{i}_{2}}$ converges as the string length increases.

\section{CONCLUSions And Future DiRections}

In this paper we have discussed string stability for a homogeneous string of strictly proper feedback control systems with nearest neighbour communications when using only linear systems with two integrators in the open loop. We have shown how the induced $L_{2}$-norm of the disturbance to error transfer function $H_{\mathrm{e}, \mathrm{d}}$ grows as the string length increases if no or a small time headway is used. A formula for the infimal time headway has been derived. We proved that using a sufficiently large time headway bounds the induced $L_{2}$ norm of $H_{\mathrm{e}, \mathrm{d}}$ independently of the string length.

As for future directions, it would be interesting to extend the results presented to more general cases. That could be analyzing heterogeneous systems, bidirectional controller designs, or using the $L_{\infty}$-norm.

\section{APPENDIX}

We will prove that an interconnected system where the maximum of (12) is achieved at $\omega=0$ is string unstable if the time headway $h$ is equal $h_{0}$ in (13). More precisely, we will show that there exists a $\tau>0$ and a $c$ such that $\left\|H_{\mathrm{e}, \mathrm{d}}\right\|_{\mathrm{i}_{2}}^{2} \geq \tau(N+c)$.

We assume that there exist a $\omega_{0} \in(0,1]$, a $l_{\text {min }}$ and a $l_{\max }$ such that $0<l_{\min } \leq|\tilde{L}(j \omega)| \leq l_{\max }$ for all $\omega \in\left[0, \omega_{0}\right)$. Then there exist $\alpha$ and $\beta$ such that $|\Gamma|^{2} \geq \frac{1}{1+\alpha \omega^{4}}$ and $\left|Q^{-1}-\Gamma\right|^{2} \geq \frac{\omega^{4}}{\beta}$ are satisfied for all frequencies $|\omega|<\omega_{0}$. Later, these inequalities will be used to prove string instability.

First, we will analyse $|\Gamma|^{2}$ for this special case.

$$
\begin{aligned}
& |\Gamma|^{2}=\frac{1}{h^{2} \omega^{2}+1} \frac{\frac{1}{\omega^{4}}|\tilde{L}|^{2}}{1-\frac{1}{\omega^{2}}(\tilde{L}+\tilde{\tilde{L}})+\frac{1}{\omega^{4}}|\tilde{L}|^{2}} \\
& =\frac{1}{\frac{2}{|\tilde{L}(0)|} \omega^{2}+1} \frac{|\tilde{L}|^{2}}{\omega^{4}-\omega^{2}(\tilde{L}+\tilde{\tilde{L}})+|\tilde{L}|^{2}} \\
& =\left(\frac{2}{|\tilde{L}(0)||\tilde{L}|^{2}} \omega^{6}+\frac{\omega^{4}}{|\tilde{L}|^{2}}-\frac{2}{|\tilde{L}(0)|} \frac{\tilde{L}+\overline{\tilde{L}}}{|\tilde{L}|^{2}} \omega^{4}\right. \\
& \left.-\frac{\tilde{L}+\overline{\tilde{L}}}{|\tilde{L}|^{2}} \omega^{2}+\frac{2}{|\tilde{L}(0)|} \omega^{2}+1\right)^{-1}
\end{aligned}
$$

We want to find an $\alpha$ such that $|\Gamma|^{2} \geq \frac{1}{1+\alpha \omega^{4}}$ for small frequencies $|\omega|<\omega_{0}$. Hence, $\alpha$ must satisfy

$$
\begin{aligned}
\alpha \geq & \sup _{|\omega|<\omega_{0}}\left(\frac{2}{|\tilde{L}(0)||\tilde{L}|^{2}} \omega^{2}+\frac{1}{|\tilde{L}|^{2}}-\frac{2}{|\tilde{L}(0)|} \frac{\tilde{L}+\overline{\tilde{L}}}{|\tilde{L}|^{2}}\right. \\
& \left.+\frac{-\frac{\tilde{L}+\tilde{\tilde{L}}}{|\tilde{L}|^{2}}+\frac{2}{|\tilde{L}(0)|}}{\omega^{2}}\right)
\end{aligned}
$$

For all fixed frequencies $0<|\omega|<\omega_{0}$, there exists a $\alpha$ which satisfies (26). However, it also must be bounded for $\omega \rightarrow 0$.

$$
\begin{gathered}
\alpha \geq \lim _{\omega \rightarrow 0}\left(\frac{2}{|\tilde{L}(0)||\tilde{L}|^{2}} \omega^{2}+\frac{1}{|\tilde{L}|^{2}}-\frac{2}{|\tilde{L}(0)|} \frac{\tilde{L}+\overline{\tilde{L}}}{|\tilde{L}|^{2}}\right. \\
\left.+\frac{-\frac{\tilde{L}+\tilde{\tilde{L}}}{|\tilde{L}|^{2}}+\frac{2}{|\tilde{L}(0)|}}{\omega^{2}}\right)
\end{gathered}
$$

To evaluate the last term in (27), we make use of the following facts:

$$
\begin{aligned}
\tilde{L}(\omega) & =a(\omega)+b(\omega) j \\
a(\omega) & =a_{0}+a_{2} \omega^{2}+a_{4} \omega^{4}+\ldots \\
b(\omega) & =b_{1} \omega+b_{3} \omega^{3}+b_{5} \omega^{5}+\ldots
\end{aligned}
$$

and L'Hôpital's Rule:

$$
\begin{aligned}
& \lim _{\omega \rightarrow 0} \frac{-\frac{\tilde{L}+\overline{\tilde{L}}}{|\tilde{L}|^{2}}+\frac{2}{\omega^{2}}}{|\tilde{L}(0)|} \\
& =\lim _{\omega \rightarrow 0} \frac{-(\tilde{L}+\tilde{\tilde{L}})+\frac{2}{|\tilde{L}(0)|}|\tilde{L}|^{2}}{|\tilde{L}|^{2} \omega^{2}} \\
& =\lim _{\omega \rightarrow 0} \frac{-\frac{\mathrm{d}}{\mathrm{d} \omega}(\tilde{L}+\tilde{\tilde{L}})+\frac{2}{|\tilde{L}(0)|} \frac{\mathrm{d}}{\mathrm{d} \omega}\left(|\tilde{L}|^{2}\right)}{\frac{\mathrm{d}}{\mathrm{d} \omega}\left(|\tilde{L}|^{2}\right) \omega^{2}+2|\tilde{L}|^{2} \omega} \\
& =\lim _{\omega \rightarrow 0} \frac{-\frac{\mathrm{d}^{2}}{\mathrm{~d} \omega^{2}}(\tilde{L}+\overline{\tilde{L}})+\frac{2}{\mid \tilde{L}(0)} \frac{\mathrm{d}^{2}}{\mathrm{~d} \omega^{2}}\left(|\tilde{L}|^{2}\right)}{\frac{\mathrm{d}^{2}}{\mathrm{~d} \omega^{2}}\left(|\tilde{L}|^{2}\right) \omega^{2}+4 \frac{\mathrm{d}}{\mathrm{d} \omega}\left(|\tilde{L}|^{2}\right) \omega+2|\tilde{L}|^{2}} \\
& =\frac{-4 a_{2}+\frac{2}{\left|a_{0}\right|}\left(4 a_{0} a_{2}+2 b_{1}^{2}\right)}{2 a_{0}^{2}}
\end{aligned}
$$

Since $\tilde{L}(0)=a_{0} \neq 0$, the limit in (31) exists. Therefore (27) is bounded, and there exists an $\alpha$ that satisfies (26) for all frequencies $|\omega|<\omega_{0}$ and

$$
|\Gamma|^{2} \geq \frac{1}{1+\alpha \omega^{4}} \quad \forall|\omega|<\omega_{0}
$$


We will now show that there exists a $\beta$ satisfying $\left|Q^{-1}-\Gamma\right|^{2} \geq \frac{\omega^{4}}{\beta}$.

$$
\left|Q^{-1}-\Gamma\right|^{2}=\frac{1}{h^{2} \omega^{2}+1} \frac{\omega^{4}}{\omega^{4}-\omega^{2}(\tilde{L}+\overline{\tilde{L}})+|\tilde{L}|^{2}}
$$

For small frequencies $|\omega|<\omega_{0}$, there exists a $\beta^{\prime}$ such that

$$
h^{2} \omega^{2}+1 \leq h^{2}+1=\beta^{\prime} \quad \forall|\omega|<\omega_{0}
$$

Furthermore, there exists a $\beta^{\prime \prime}$ satisfying

$$
\omega^{4}-\omega^{2}(\tilde{L}+\overline{\tilde{L}})+|\tilde{L}|^{2} \leq \beta^{\prime \prime} \quad \forall|\omega|<\omega_{0}
$$

such that

$$
\beta^{\prime \prime}=1+\sup _{|\omega|<\omega_{0}}\left(|\tilde{L}+\overline{\tilde{L}}|+|\tilde{L}|^{2}\right)
$$

Hence,

$$
\left|Q^{-1}-\Gamma\right|^{2} \geq \frac{\omega^{4}}{\beta^{\prime} \beta^{\prime \prime}}=\frac{\omega^{4}}{\beta} \quad \forall|\omega|<\omega_{0}
$$

Using the special structure of $H_{\mathrm{e}, \mathrm{d}}$, we can bound its induced $L_{2}$-norm as follows:

$$
\begin{aligned}
\left\|H_{\mathrm{e}, \mathrm{d}}\right\|_{\mathrm{i}_{2}}^{2}+\left\|\Gamma C^{-1}\right\|_{\mathrm{i}_{2}}^{2} & \geq\left\|H_{\mathrm{e}, \mathrm{d}}+I_{N} \Gamma C^{-1}\right\|_{\mathrm{i}_{2}}^{2} \\
& =\sup _{\|v\|=1}\left\|\left(H_{\mathrm{e}, \mathrm{d}}+I_{N} \Gamma C^{-1}\right) v\right\|_{\mathrm{i}_{2}}^{2} \\
& \geq\left\|\left(H_{\mathrm{e}, \mathrm{d}}+I_{N} \Gamma C^{-1}\right) v^{\prime}\right\|_{\mathrm{i}_{2}}^{2}
\end{aligned}
$$

with a vector $v^{\prime}$ of length 1

$$
v^{\prime}=\frac{1}{\sqrt{N}}\left(1 \frac{\Gamma}{\|\Gamma\|} \frac{\Gamma^{2}}{\|\Gamma\|^{2}} \cdots \frac{\Gamma^{N-1}}{\|\Gamma\|^{N-1}}\right)^{\mathrm{T}}
$$

Using $|\Gamma| \leq 1 \forall \omega$, (32), (37) and (39), inequality (38) becomes

$$
\begin{aligned}
\left\|H_{\mathrm{e}, \mathrm{d}}\right\|_{\mathrm{i}_{2}}^{2}+ & \left\|\Gamma C^{-1}\right\|_{\mathrm{i}_{2}}^{2} \\
\geq & \operatorname{ess} \sup _{\omega \in \mathbb{R}} \frac{\left|Q^{-1}-\Gamma\right|^{2}|\Gamma|^{2}\left|C^{-1}\right|^{2}}{N}\left(1+(1+|\Gamma|)^{2}+\cdots\right. \\
& \left.\cdots+\left(1+|\Gamma|+|\Gamma|^{2}+\cdots+|\Gamma|^{N-2}\right)^{2}\right) \\
\geq & \operatorname{ess} \sup _{|\omega|<\omega_{0}} \frac{\left|C^{-1}\right|^{2}}{N} \frac{\omega^{4}}{\beta}\left(|\Gamma|^{2 N}+\left(|\Gamma|^{N}+|\Gamma|^{N}\right)^{2}+\cdots\right. \\
& \left.\quad \cdots+\left(|\Gamma|^{N}+|\Gamma|^{N}+|\Gamma|^{N}+\cdots+|\Gamma|^{N}\right)^{2}\right) \\
\geq & \operatorname{ess} \sup _{|\omega|<\omega_{0}} \frac{\left|C^{-1}\right|^{2}}{N} \frac{\omega^{4}}{\beta}|\Gamma|^{2 N} \\
& \cdot\left(1^{2}+2^{2}+\cdots(N-1)^{2}\right) \\
\geq & \operatorname{ess} \sup _{|\omega|<\omega_{0}} \frac{\left|C^{-1}\right|^{2}}{N} \frac{\omega^{4}}{\beta} \frac{1}{\left(1+\alpha \omega^{4}\right)^{N}} \\
& \quad \frac{(N-1) N(2 N-1)}{6}
\end{aligned}
$$

For any string length $N$, the maximum over all frequencies in (40) must be greater or equal to that obtained by choosing $\omega=N^{-1 / 4}$ :

$$
\begin{aligned}
& \left\|H_{\mathrm{e}, \mathrm{d}}\right\|_{\mathrm{i}_{2}}^{2}+\left\|\Gamma C^{-1}\right\|_{\mathrm{i}_{2}}^{2} \\
& \quad \geq \frac{\left|C^{-1}\right|^{2}}{6 \beta}\left(1+\frac{\alpha}{N}\right)^{-N} \frac{(N-1) N(2 N-1)}{N^{2}}
\end{aligned}
$$

for sufficiently large strings, $N>\omega_{0}^{-4}$.

Since $\left(1+\frac{\alpha}{N}\right)^{-N} \geq \mathrm{e}^{-\alpha}$, (41) can be bounded by

$$
\begin{aligned}
\left\|H_{\mathrm{e}, \mathrm{d}}\right\|_{\mathrm{i}_{2}}^{2}+\left\|\Gamma C^{-1}\right\|_{\mathrm{i}_{2}}^{2} & \\
& \geq \frac{\left|C^{-1}\right|^{2}}{6 \beta} \mathrm{e}^{-2 \alpha} \frac{(N-1) N(2 N-1)}{6} \\
& \geq \frac{\left|C^{-1}\right|^{2}}{3 \beta} \mathrm{e}^{-2 \alpha}(N-2) \quad \forall N \geq 1
\end{aligned}
$$

Thus, the induced $L_{2}$-norm of $H_{\mathrm{e}, \mathrm{d}}$ grows at least as far as the square root of the string length $N$ and the system is not string stable according to Definition 1.

\section{REFERENCES}

[1] W. Levine and M. Athans, "On the optimal error regulation of a string of moving vehicles," IEEE Transactions on Automatic Control, vol. AC-11, no. 3, pp. 355-361, 1966.

[2] K. C. Chu, "Decentralized control of high-speed vehicular strings," Transportation Science, vol. 8, no. 4, pp. 361 - 384, 1974.

[3] S. Sheikholeslam and C. Desoer, "Longitudinal control of a platoon of vehicles," in Proceedings of the American Control Conference, 1990, pp. 291-297.

[4] D. Swaroop, "String stability of interconnected systems: An application to platooning in automated highway systems," Ph.D. dissertation, University of California, Berkeley, CA, 1994.

[5] S. Klinge, "Stability issues in distributed systems of vehicle platoons," Master's thesis, Otto-von-Guericke-University Magdeburg, http://www.hamilton.ie/publications.htm, 2008.

[6] D. Swaroop and J. Hedrick, "String stability of interconnected systems," IEEE Transactions on Automatic Control, vol. 41, no. 3, pp. 349 - 357, 1996.

[7] P. Seiler, A. Pant, and K. Hedrick, "Disturbance propagation in vehicle strings," IEEE Transactions on Automatic Control, vol. 49, no. 10, pp. $1835-1841,2004$

[8] I. Lestas and G. Vinnicombe, "Scalability in heterogeneous vehicle platoons," in Proceedings of the American Control Conference, 2007, pp. 4678-4683.

[9] J. Eyre, D. Yanakiev, and I. Kanellakopoulos, "A simplified framework for string stability analysis of automated vehicles," Vehicle Systems Dynamic, vol. 30, no. 5, pp. 375-405, 1998.

[10] P. Barooah and J. Hespanha, "Error amplification and disturbance propagation in vehicle strings with decentralized linear control," in Proceedings of the 44th IEEE Conference on Decision and Control, and the European Control Conference 2005, 2005, pp. 4964-4969.

[11] J. H. Davis and B. Barry, "A distributed model for stress control in multiple locomotive trains," Applied Mathematics \& Optimization, vol. 3, no. 2-3, pp. 163-190, June 1976.

[12] P. Barooah, P. Mehta, and J. Hespanha, "Decentralized control of vehicular platoons: Improving closed loop stability by mistuning," 2009, to appear in the IEEE Transactions on Automatic Control.

[13] C. Chien and P. Ioannou, "Automatic vehicle following," in Proceedings of the American Control Conference, 1992, pp. 1748-1752.

[14] M. Khatir and E. Davison, "Bounded stability and eventual string stability of a large platoon of vehicles using non-identical controllers," in Proceedings of the 43rd Conference on Decision \& Control, 2004, pp. 1111-1116.

[15] D. Yanakiev and I. Kanellakopoulos, "Nonlinear spacing policies for automated heavy-duty vehicles," IEEE Transactions on Vehicular Technology, vol. 47, no. 4, pp. 1365-1377, 1998.

[16] R. A. Horn and C. R. Johnson, Matrix Analysis. Cambridge University Press, 1985. 